\title{
Verrucous carcinoma of vulva associated with lichen sclerosus and condyloma: case report
}

\author{
Victor Hugo de Oliveira Ribeiro 1, *, Nilma Antas Neves 1 \\ ${ }^{1}$ Department of Gynecology, Obstetrics and Human Reproduction, Federal University of Bahia, Salvador, BA, \\ Brazil. \\ *Corresponding author: Victor Hugo de Oliveira Ribeiro. Humberto de Campos st., 256 - Graça. Zip Code: 40.150- \\ 130 - Salvador, BA, Brazil. Phone: +55 (71) 9 9190-4494. E-mail: ribeirovhugo@gmail.com / \\ ribeiro.victor@ebserh.gov.br.
}

Research Ethics Committee Approval: Decision n. 4.261.645 / CAAE: 36857220.6.0000.0049.

Received on: Nov 4, 2021. Accepted on: Nov 14, 2021. Available online: Nov 15, 2021.

\begin{abstract}
Vulvar Verrucous Carcinoma (VVC) is a rare lesion, with few described cases. It has low metastatic potential with high morbidity due to the necessity of extensive resections, although. Previously, VVC was considered a synonym to the BuschkeLowenstein Tumor (BLT) or Giant Condyloma Acuminatum (GCA). Lichen Sclerosus (LS) is associated with Vulvar Intraepithelial Neoplasia (VIN) and Vulvar Squamous-cell carcinoma (SCC); association with VVC is also described. The case of a 60-year-old menopausal woman is reported; she had chronic itching and an extensive verrucous lesion in vulva, initially diagnosed and treated as condyloma acuminatum; there was recurrence as verrucous carcinoma associated to LS. Excision with margins was performed and clobetasol and imiquimod were used. Patient had complete remission with no further recurrences. Distinction between VVC and BLT can be difficult; current literature considers them different entities. Human papillomavirus (HPV) infection and the presence of LS play a controversial role in these injuries.
\end{abstract}

Keywords: Verrucous Carcinoma; Vulvar Lichen Sclerosus; Condylomata Acuminata; Bushcke-Lowenstein Tumor.

\section{Introduction}

In 1948, Ackerman described the first case of verrucous carcinoma (VC) as a variant of a squamous-cell carcinoma (SCC), in oral cavity [1]. There are descriptions of $\mathrm{VC}$ in other anatomical sites. Kraus and Pérez-Mesa (1966) did the first report of vulvar verrucous carcinoma (VVC) [2]. VC is 
considered a rare disease; occurrence records consist of some series and case reports [3-5].

Even though some authors define it as a vulvar SCC (VSCC) variant, VVC presents some particular clinical characteristics; it has locally aggressive behavior, with extensive injuries and high recurrence rates, although its growth is slow and it has low potential to generate nodal or distant metastases $[4,5]$. Because of their ample extension, VVC lesions occasionally demand extensive resections, sometimes requiring grafting, and leading to inevitable loss of functionality and esthetical damage [3].

These characteristics make VVC diagnosis to be sometimes mistaken for other entities, mainly the BuschkeLowenstein Tumor (BLT) - or Giant Condyloma Acuminatum (GCA) - and Vulvar SCC itself. BLT is a benign, uncommon lesion, occurring more frequently in immunosuppressed individuals, with strong association with HPV infection (a variant of condyloma acuminata) [6].

Lichen Sclerosus (LS) is a chronic, progressive, inflammatory dermatosis, that more commonly affects the anogenital region, occurring mostly in perimenopausal women $[7,8]$. Although benign, LS is associated with differentiated-type vulvar intraepithelial neoplasia (dVIN) and with vulvar cancer. VSCC is the main type of vulvar cancer associated with LS [9].

Due to its low frequency, VVC has since appeared in the literature only through small series and case reports, with less than 100 cases described [4].
Much is discussed about the role of $\mathrm{HPV}$ and HPV-associated lesions in the pathogenesis of VVC; however, this process is still poorly understood; still, records of VVC and LS association are equally scarce. Accumulation of evidence over such cases will allow filling the gaps regarding VVC pathogenesis and its clinical behavior.

\section{Case report}

We report the case of a 60-yearold woman, menopause at the age of 47 , with no previous reports of sexually transmitted infections (STIs); she had no sexual activity since the age of 43 . For 12 months she had presented with vulvar pruritus and progressive growth of a verrucous lesion in the right hemivulva, being diagnosed with condyloma acuminatum and LS, treated with clobetasol propionate at $0.05 \%$ and imiquimod cream at $5 \%$ for 04 weeks, by an assistant gynecologist in her hometown.

In the next three months, however, the right vulvar verrucous lesion started to grow again, quite pruritic, when she came to our service. Upon examination, there was an elongated tumoral lesion measuring approximately $5 \mathrm{~cm}$ in its largest diameter, with a hypochromic, scaly, slightly moist surface and with a foul odor (Figure 1a). Furthermore, it was possible to notice a mild effacement of vulvar labia. It was made a clinical suspicion of GCA or BLT. A seven-day oral treatment was started with oral metronidazole and local hygiene of the lesion with boric water, after which the 
tumor was excised. Histopathological evaluation revealed an exophytic lesion measuring $5.0 \times 3.5 \times 2.0 \mathrm{~cm}$, with papillomatosis, hyperkeratosis, acanthosis and koilocytosis organized around deep fibrovascular axes under microscopy, with the diagnosis of condyloma acuminatum (Figure 1b). Imiquimod was reintroduced and topical hydrocortisone at $1 \%$ was added due to pruritus. HPV molecular test was not available.
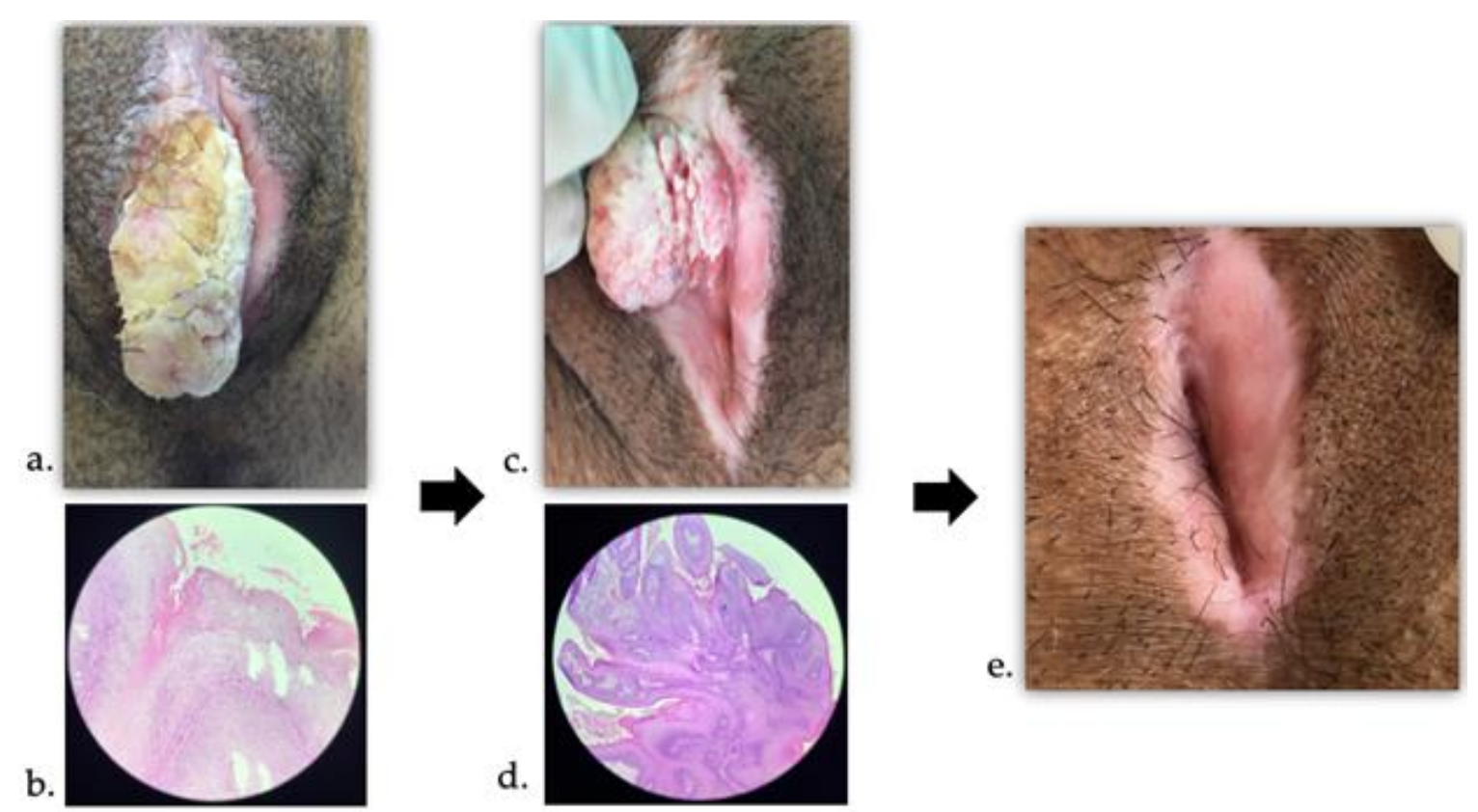

Figure 1. Macro and microscopic aspects of the vulvar lesion. At presentation, (a) verrucous elongated lesion in right hemivulva, desquamative, umid, smelling, with (b) its microscopic aspect. Then, (c) recurrence as a verrucous lesion, with (d) its microscopic aspect. Finally, (e) appearance of the vulva 30 months after the onset of the first signs and symptoms.

After two months, the patient returns with recurrence of right verrucous lesion and worsening of pruritus. The aspect was of verruciform tumor, with papillae, located on the right hemivulva, and an area of thickening over a hypochromic patch in the same side, slightly more cranial (Figure 1c). Exeresis of the verrucous lesion was performed, with margins, and also patch-biopsy of the thickening area, whose histopathology revealed, respectively: Vulvar Verrucous
Carcinoma (reported, by pathologist, as a synonym to BLT), well-differentiated from $3.5 \times 2.5 \times 1.5 \mathrm{~cm}$, with free margins, containing acanthosis and rare koilocytes - although the tumor-dermis interface showed an expansive pattern, there were no signs of microinvasion or perineural or angiolymphatic invasion (Figure 1d); and lichen sclerosus with areas of papillomatous acanthosis. Imiquimod was maintained, topical corticosteroid was modified to clobetasol, and a computed tomography 
scan of the pelvis was done, which did not observe tumor lesions or lymphadenomegaly, with normal results.

The patient was kept under bimonthly follow-up for six months after the second biopsy, using imiquimod and clobetasol, starting weaning later, keeping only corticosteroids on demand. Thirty months from the onset of the first symptoms, the pruritus evolved with complete improvement; clinical examination of the vulva showed introital narrowing, complete effacement of labia, some hypochromia, but no new tumor lesions or thickening (Figure 1e). Clinical follow-up was carried out quarterly and, subsequently, every six months. Finally, throughout the follow-up, four cervical cytology exams were performed, every six months, all negative for malignancy. Colposcopy was normal in all assessments.

\section{Discussion and Conclusion}

The clinical and histopathological distinction between BuschkeLowenstein Tumor (BLT) or Giant Condyloma Acuminata (GCA) and Verrucous Carcinoma (VC) can be difficult, especially in the context of Lichen Sclerosus (LS), in which the epithelial changes that occur are common to BLT and VC [6,9-10].

BLT is a benign lesion associated with HPV infection (mainly subtypes 6 and 11) that occurs primarily in younger women; macroscopically it behaves as an exuberant and locally aggressive tumor; the microscopic appearance, however, is of a benign lesion, with acanthosis, hyperkeratosis and koilocytosis; there are long fibrovascular cores upon which the endophytic component of the lesion is organized $[9,12]$. VC, on the other hand, has shorter fibrovascular cores, the aspect of a well-differentiated squamous cell carcinoma with little or no koilocytosis and the tumor-dermis interface demonstrating a pushing, noninfiltrative aspect. Most patients are in post-menopause [6].

The relationship between VC and HPV is controversial. Crowther (1988) demonstrated, in a VC case, the presence of subtype 11; in this pioneer report there is some fragility, however, in the diagnostic criteria for $\mathrm{VC}$ and the methodology for HPV detection [13]. A relationship between VVC and chronic inflammatory conditions such as LS, with or without dVIN, has been described $[3,4,11]$.

Wang et al. (2010) reported 13 cases of VC, five of which were of vulvar origin; Molecular testing for HPV was only possible for two patients with $\mathrm{VVC}$, one of them being positive for HPV subtype 11 - all had lichen sclerosus, with clinical appearance of verrucous lesions emerging from the skin with a lichenified aspect, with hypochromia and pruritus. Derrick et al. (2000) report two cases of VVC: both had histological evidence of LS associated with VVC [14]. Nascimento et al. (2004) attribute to epithelial acanthosis a possible role as a common 
precursor for the emergence of $\mathrm{dVIN}$ or VVC in the context of LS [15].

Liu et al. (2015) report six cases of VVC - in three of them there was an initial misdiagnosis of BLT; no HPV test was performed. There were no recurrences. VSCC was found concomitantly in one of the cases; dVIN, in two. They also reviewed reports and case series published between 1994 and 2014 in Chinese and English publications [11]. In Chinese studies, 20 cases had been reported; the mean age of the patients was 62 years and $80 \%$ of them were postmenopausal. Three patients had a diagnosis of lichen; two patients had simultaneous condyloma acuminatum. Four patients underwent hybrid capture for HPV, with positive results for three of them (subtypes 6 and 11). In three cases, an initial misdiagnosis of BLT was reported. In Western literature, on the other hand, there were 41 cases reported in the period, in patients aged 32 to 96 years; HPV molecular test was available for 16 (39\%) cases $-6(38 \%)$ were positive [11]. Zhang et al. (2019), using data from the PubMed database on VVC cases, reviewed a total of 50 cases in 12 publications between 1988 and 2018. HPV genotyping was performed in four publications (18 patients): one patient tested positive (for the high-risk oncogenic subgroup). In most of the reviewed studies, the presence of some form of lichen was reported among the cases [4].

The pathophysiological mechanism behind VVC is still unclear. Gualco et al. (2003) reported ten cases of VVC; the presence of HPV was not demonstrated in any of the cases using in situ hybridization. The authors also evaluated the immunohistochemical pattern of these lesions using keratin AE1, which was positive in surface and intermediate layers, and AE3, positive in basal layer. The cases were negative for wild and mutant types of p53 protein [5]. On the other hand, Derrick et al. (2000) and Wang et al. (2010) propose that LS predisposes the vulvar epithelium to the onset of VVC through the mechanisms of chronic inflammation, altered p53 expression and oxidative stress [14]; the second paper also implies a possible role of $\mathrm{HPV}$ infection in the emergence of VVC in the LS context [3].

There is no consensus on the best treatment for VVC. There seems to be a tendency to perform surgical resection with margins (including partial and radical vulvectomy), with or without lymph node management, followed by strict clinical follow-up due to the potential for recurrence ${ }^{(12)}$. Studies on VVC or VSCC do not mention the use of imiquimod as adjuvant therapy, as it was done with this patient.

There are no described cases of VVC arising as a recurrence of condyloma in association with LS so far. As in current literature, this case points to: the diagnostic difficulties (clinical and histopathological); the limited information available on molecular diagnosis of HPV; the concomitance with the diagnosis of LS; the option of treatment with surgical excision, leading to an almost inevitable functional and aesthetic loss. The use of imiquimod as 
an adjuvant to the surgical treatment could be a promising field of research.

\section{References}

[1] Ackerman LV. Verrucous carcinoma of the oral cavity. Surgery. 1948 Apr;23(4):670-8.

[2] Kraus FT, Pérez-Mesa C. Verrucous carcinoma. Clinical and pathologic study of 105 cases involving oral cavity, larynx and genitalia. Cancer. 1966 Jan;19(1):26-38. doi: 10.1002/10970142(196601)19:1<26:aidcncr2820190103> 3.0.co;2-1.

[3] Wang SH, Chi CC, Wong YW, Salim A, Manek S, Wojnarowska F. Genital verrucous carcinoma is associated with lichen sclerosus: a retrospective study and review of the literature. J Eur Acad Dermatol Venereol. 2010 Jul;24(7):815-9. doi: 10.1111/j.1468-3083.2009.03531.x.

[4] Zhang W, Wang Y, Chen W, Du J, Xiang L, Ye S, Yang H. Verrucous Carcinoma of the Vulva: A Case Report and Literature Review. Am J Case Rep. 2019 Apr 19;20:551-556. doi: 10.12659/AJCR.914367.

[5] Gualco M, Bonin S, Foglia G, Fulcheri E, Odicino F, Prefumo F, Stanta G, Ragni N. Morphologic and biologic studies on ten cases of verrucous carcinoma of the vulva supporting the theory of a discrete clinico-pathologic entity. Int J Gynecol Cancer. 2003 MayJun;13(3):317-24 . doi: 10.1046/j.15251438.2003.13200.x.

[6] Bambao C, Nofech-Mozes S, Shier M. Giant condyloma versus verrucous carcinoma: a case report. J Low Genit
Tract Dis. 2010 Jul;14(3):230-3. doi: 10.1097/LGT.0b013e3181c945ed.

[7] Powell JJ, Wojnarowska F. Lichen sclerosus. Lancet. 1999 May 22;353(9166):1777-83. doi: 10.1016/s01406736(98)08228-2.

[8] van de Nieuwenhof HP, Bulten J, Hollema H, Dommerholt RG, Massuger LF, van der Zee AG, de Hullu JA, van Kempen LC. Differentiated vulvar intraepithelial neoplasia is often found in lesions, previously diagnosed as lichen sclerosus, which have progressed to vulvar squamous cell carcinoma. Mod Pathol. 2011 Feb;24(2):297-305. doi: 10.1038/modpathol.2010.192.

[9] Elsayed AG, Sola-Rufai ST, Griswold D, Pacioles T. Verrucous carcinoma arising in a long standing BuschkeLöwenstein tumor. Clin Case Rep. 2019 Feb 19;7(4):836-838. doi: 10.1002/ccr3.2029.

[10] Ghaemmaghami F, Nazari Z. Giant condyloma accuminatum mimicking vulvar verrucous carcinoma. Eur J Surg Oncol. 2007 Jun;33(5):668-9. doi: 10.1016/j.ejso.2006.09.031.

[11] Liu G, Li Q, Shang X, Qi Z, Han C, Wang Y, Xue F. Verrucous Carcinoma of the Vulva: A 20 Year Retrospective Study and Literature Review. J Low Genit Tract Dis. 2016 Jan;20(1):114-8. doi: 10.1097/LGT.0000000000000164.

[12] Campaner, AB, Cardoso FA, Fernandes GL, Veasey JV. Verrucous carcinoma of the vulva: diagnosis and treatment. Anais Brasileiros de Dermatologia [online]. 2017;92(2):243245. doi:10.1590/abd1806-4841.20174929. 
[13] Crowther ME, Shepherd JH, Fisher

C. Verrucous carcinoma of the vulva containing human papillomavirus-11. Case report. Br J Obstet Gynaecol. 1988 Apr;95(4):414-8. doi: 10.1111/j.14710528.1988.tb06617.x.

[14] Derrick EK, Ridley CM, KobzaBlack A, McKee PH, Neill SM. A clinical study of 23 cases of female anogenital carcinoma. Br J Dermatol. 2000 Dec;143(6):1217-23. doi: 10.1046/j.13652133.2000.03891.x.

[15] Nascimento AF, Granter SR, Cviko A, Yuan L, Hecht JL, Crum CP. Vulvar acanthosis with altered differentiation: a precursor to verrucous carcinoma? Am J Surg Pathol. 2004 May;28(5):638-43. doi: 10.1097/00000478-200405000-00012.

Conflict of interest: The author declares no conflicts of interest.

Funding: No external financial support was received for this research. The case report was carried out with authors' own resources.

How to cite this article: Ribeiro VHO, Neves NA. Verrucous carcinoma of vulva associated with lichen sclerosus and condyloma: case report. Brazilian Journal of Case Reports. 2021 OctDec;01(4):153-159. 